\title{
Research on Pupil Segmentation and Localization in Micro Operation
}

$$
\text { Hu BinLiang }{ }^{1, a} \text {, Chen GuoLiang, }{ }^{2, ~} \text {, Ma Hui }{ }^{2, c}
$$

${ }^{1}$ School of Mechanical and Electronic Engineering, Hunan University of Science and Technology, Xiangtan 411201, Hunan, China

\author{
${ }^{2}$ School of Mechanical and Electronic Engineering, Wuhan University of Technology, Wuhan, \\ 430070, China \\ ${ }^{2}$ School of Mechanical and Electronic Engineering, Wuhan University of Technology, Wuhan, \\ 430070, China \\ aemail: blhu@hnust.edu.cn, bemail: glchen@whut.edu.cn, cemail: mahui_career@163.com
}

\section{Keywords: Micro Operation; Pupil Localization; Hough Transform}

\begin{abstract}
In this paper, a fast pupil segmentation and localization algorithm is proposed owing to the characteristics that the operator's eyeball deflects slightly and the gray level of eye image changes a little. Firstly, in order to segment high quality pupil region, we segment the specific interest region, use down sampling method to reduce the calculated pixels, apply the 8-connected components to the binary image denoising and use line scanning method to amend the light spot. Then, the canny operator is applied to detect the edge of pupil region. Finally, we take Hough transform to locate pupil center. The experimental results show that the new algorithm can segment high quality pupil region, locate the pupil center and is applicable to pupil image captured by head-mounted device.
\end{abstract}

\section{Introduction}

Pupil localization is an important research topic in computer vision field, which is used in human-computer interaction, traffic safety, artificial intelligence, medical and other fields widely. Recently, corresponding pupil orientation methods are proposed by many scholars aiming at the pupil image characteristics in different applications. Aiming at head-mounted eye tracking systems and allowing for the different shade degrees, $\mathrm{Pu}$ et al. [1] proposed the classified pupil localization algorithm, which ensured speed and precision. Based on the image characteristics of fundus camera, Chen et al. [2] proposed a fast pupil segmentation algorithm, employing a variety of methods. Experiment results showed that the algorithm is robust and fast. On the basis of the pupil gray level and geometric feature, Yan et al. [3] achieved precise locating in infrared and visible light combined mathematical morphology and Hough transform. Li et al. [4] put forward a hybrid algorithm for video-based eye tracking combining feature-based methods and model-based methods. To solve the problems caused by distortion and disturbance, a new Random Sample Consensus algorithm based on only 3 points, named RANSAC_3, is proposed by Zhang et al. [5]. According to the videonystagmograph, Wang et al. [6] proposed an algorithm locating the eye pupil center by the coarse locating iris center with gray clustering.

Based on the characteristics that the operator's eyeball deflects slightly and the gray level of eye image changes a little, the segmentation and localization method of operator pupil was studied in this paper through using computer vision and image processing technology, which is suited to micro operation technology and its environmental features. Consequently, the micro operation systems will have capabilities to obtain and then locate the operator's interest region automatically, and lay foundation of realization of automatic micro operations. 


\section{The Main Characteristics of the Pupil Image}

Due to the structure of head-mounted device in the experiment, some interference information, such as eyebrow and the surrounding, may appear in the image. Head-mounted device is shown in Figure 1.

The picture taken by head-mounted device is shown in Figure 2 (a), the proportion of eye region in this picture is relatively small. If the pupil localization is carried out directly in acquired image, it will cause the algorithm complicated and the loss of locating accuracy because of noise. As shown in Figure 1, on the condition that the head-mounted device is fixed, the relative position of the camera and eye is stationary. Therefore, the eye is mainly concentrated in fixed-size rectangular region, as shown in Figure 2 (b). Meanwhile, owing to the constancy of micro operations surrounding, which the most important factor is the brightness constancy of surroundings, the gray level of the image will not change.

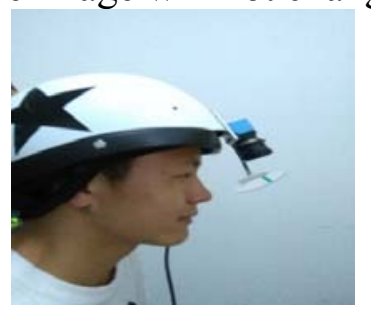

Fig.1. Head-mounted device

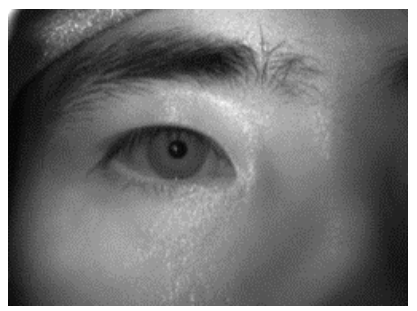

(a) Eye image

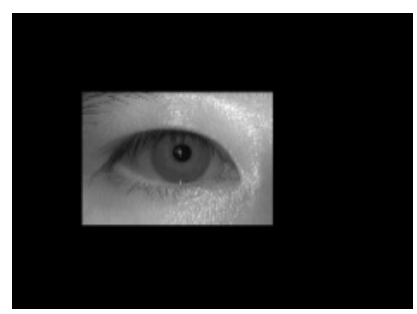

(b) Specific rectangular region

Fig.2. Eye image and specific rectangle region

The shape of pupil and covered scope vary with the different position of pupil in the eyes, which is caused by different postures of eyes. In addition, when eyes look some certain direction or squinting, parts of the eyeball will be covered by eyelids, which will make the outline of pupil captured by camera ellipse and incomplete [1] [7].

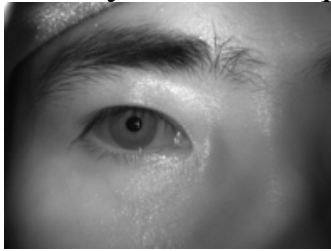

(a) Upper left corner



(b) Lower left corner

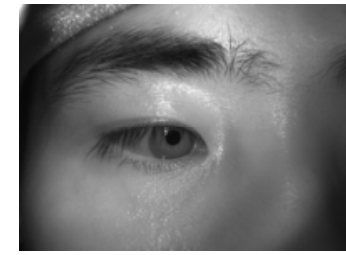

(c) Lower right corner

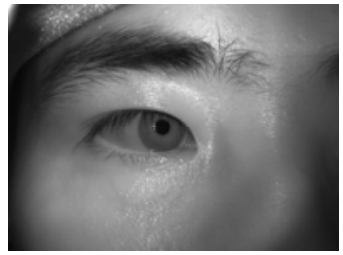

(d) Upper right corner

Fig.3. Eye image of different gaze points

On the basis of the characteristics of the micro operation, the distance between the operator and human-computer interaction interface is short, which means that the operator keep an appropriate distance from computer. As shown in Figure 3, the eye images are captured when the micro operator look at the four borders of the human-computer interaction interface. Which could be seen that eye deflection angle is smaller.

\section{The Pupil Segmentation}

According to the characteristics of pupil image captured in micro operation situation. On the basis of reference [2], this paper proposes a fast pupil segmentation algorithm use a combination of specific interest region, down sampling, stationary threshold, 8-connected components and line scanning method. The specific methods are as follows.

\section{A. Segmentation of Specific Interest Region}

In micro operation, eyeball deflection of the operator is smaller. Owing to the fixed head-mounted device, the relative position of the camera and eye will stay relatively stationary, therefore, we can segment a specific region of an image. The method of determining the specific interest region is: binarizing image with a small threshold, then locating the center of the pupil $\left(x_{0}\right.$, $\left.y_{0}\right)$ and the radius $r_{0}$ in the binary image, using the Hough transform circle detection. The specific 
algorithm is as follows.

$$
\left\{\begin{array}{c}
x=x_{0}-8 r_{0} \\
y=y_{0}-10 r_{0} \\
l=16 r_{0} \\
w=20 r_{0}
\end{array}\right.
$$

where $(x, y)$ is the upper left starting point of a rectangular region, $l, w$ is the length and width of a rectangular region respectively.

The interest region of Figure 4 is extracted from Figure 2(a). Specific region image substantially contain the prime information of the operator's eye, so that the pupil is included. It is not only reduces the recognition region of the entire image, improve the efficiency of the subsequent pupil localization through such operation, but also greatly reduced the noise interference which is caused by eyebrows and surrounding the image.

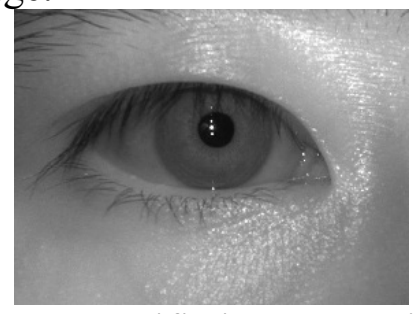

Fig.4. Specific interest region

This need to be highlighted that the specific interest region, which need to be recalculated, vary depending on different micro operators. The recalculation progress will be accomplished quickly.

\section{B. The Down Sampling}

The down sampling method is on an original sample value sequence conforming to certain sampling rules, so that a new image sequence can be obtained. The down sampling process makes the image pixel data quantity reduce exponentially and can greatly improve computing speed without loss of precision. The specific interest region image is to be processed by in (2).

$\operatorname{Out}(i, j)=\operatorname{Samp}(I(x, y))$

where $I(x, y)$ is the specific interest region, $\operatorname{Samp}()$ indicates the down sampling of the specific interest region.

$\operatorname{Samp}()=f(1: k: m, 1: k: n)$

where $m, n$ is the size of the image, $k$ is sampling rate.

Sampling rate is determined by the size of the image, the more pixels the image have, and the higher the sampling rate will be. However, the sampling rate is not the higher the better.

\section{The Acquisition of Binary Threshold}

Allowing for the characteristics of the micro-operation in a specific circumstance, the gray level of captured pupil image maintain stability and the threshold is relatively stationary. Therefore, the stationary threshold value can be used in binary process image.

The threshold is the key factor that influence the accuracy of locating, for example, low threshold value will cause the reduction of pupil section, and also high threshold will make the non-pupil section to be mistaken for the real pupil. The improved threshold algorithm is as follows based on [8].

1) Get the histogram of the image, as shown in Figure 5(a). We can see the location of peaks and valleys more apparent after median filtering in Figure 5(b). Seek the gray value T0 of the valley which between the first wave and the second wave. In most cases, we can obtain a good result by using T0. But if the gray value of the eyelash is lower than the pupil, the threshold we adopt is too low and the pupil will be recognized as background by mistake, so we need further judgment.

2) Calculate the difference between $\mathrm{T} 0$ and the gray value of the first wave, if the difference is greater than 3, the value T0 can be used as the final threshold value T, or we should seek the valley after the next wave in histogram. 
We use the method above to obtain the final threshold of ten samples images, and then calculate their average as the fixed threshold. The value of the fixed threshold is 25 . Defective pupil and noise block can be seen in the binary image in Figure 6, because light spot and the gray level of eyelashes are similar to those of pupil.

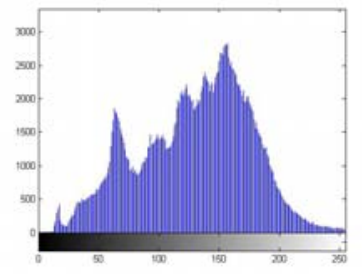

(a) Histogram



(b) Histogram after filtering

Fig.5. Sample image histogram

\section{Segmentation and Mending of Pupil}

8-connected components method [9] is used in the segmentation of pupil region, and scanning line method is used in filling the light spot from the horizontal and vertical directions. For example, a typical scanning process contain three steps as follows.

Step 1: Scanning from left to right in the horizontal direction, point $x_{1}$ stand for the first point in a row whose gray value is zero.

Step 2: Scanning from right to left, point $x_{2}$ stand for the first point in a column whose gray value is zero.

Step 3: The gray value of points in that row between point $x_{1}$ and point $x_{2}$ was set zero.

The aforementioned scanning process was applied to all rows in horizontal direction and all columns in the vertical direction repeating, so that scanning of the whole image will be completed.

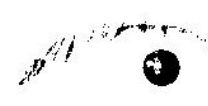

Fig.6. Binary image

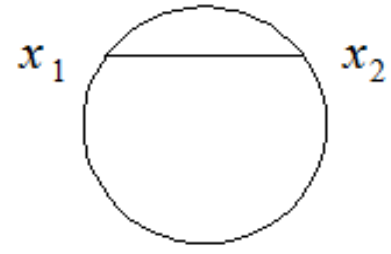

Fig.7. Line scanning method

\section{Localization of the Pupil}

In micro operation, the deflection angle of operator eyes is small, the pupil is a circle. First, we use canny operator to detect the edges of the pupil, and then locate the pupil center by Hough transform. Dr. Richard P. Wildes of US Samoff companies [10] achieved pupil localization applying Hough Transform in 1997. On the basis of the principle of Hough transform, the mapping relation between $x y$ and $\rho \theta$ in Hough transform is as follows.

The general equation of a circle in the Cartesian coordinate system is $(x-a)^{2}+(y-b)^{2}=r^{2}$

The polar equation of a circle in the $\rho \theta$ plane of polar coordinate system is

$\left\{\begin{array}{l}x=a+r \cos \theta \\ y=b+r \sin \theta\end{array}\right.$

where $a, b$ is the center coordinates; $r$ is the radius of the circle. The accumulation arrays dimension is 3 in the parameter space because of there are three parameters $a, b, r$ in image space. The points in image space $(x, y)$ are transformed to the points in parameter space $(a, b, r)$ correspondingly through Hough transform. Algorithm steps are as follows.

1) The maximum and minimum values of parameters $a, b, r(a, b$ is the center coordinates of pupil, $r$ is the radius of the pupil) of are estimated based on the size of image. The three-dimensional discrete parameter space can be set up, the size of parameter space is determined by the maximum and minimum values of parameters $a, b, r$. 
2) Setting the accumulator array $\mathrm{A}(a, b, r)$ in the parameter space and initialing the three-dimensional array.

3) Adopting Hough transform to calculate the edge points of the image. Voting the circle parameters $(a, b, r)$ which represent the edge points, adding one to the accumulator of the corresponding small cubic lattice.

4) Seeking the peak value of all accumulators, the coordinates of peak in small grid are corresponding to the center of all points that sharing the same circle in image space.

\section{Experiment and Analysis}

The source code was written in MATLAB. The experiments were performed in MATLAB 7.0 running on Windows 7.0. The PC was an Intel (R) core (TM) i3 370M 2.4GHZ processor with $2 \mathrm{~GB}$ of DDR-1333 RAM.

The range of selected parameters directly affect the result of detection. Based on the coarse location radius $r_{0}$ and the pupil center coordinates $\left(x_{0}, y_{0}\right)$ above, we can efficiently reduce the ranges of the radius and center in Hough transform. In this experiment, we make the variables $r, a, b$ and $\theta$ belong to the ranges $\left[r_{0^{-}} \delta_{1} ; r_{0}+\delta_{1}\right],\left[x_{0^{-}} \delta_{2} ; x_{0}+\delta_{2}\right],\left[y_{0}-\delta_{2} ; y_{0}+\delta_{2}\right]$ and $[0 ; 2 \pi]$ respectively. On account of the errors of the coarse location above, we expand the scope, and we make $\delta_{1}=5, \delta_{2}=10$ in this experiment. The step size of $r$ is 0.5 and the step size of $\theta$ is 0.1 . The pupil detection time will increase if the image is large or step size is small. Selecting appropriate parameter range and step size are crucial to the experiment.

Defective pupil and noise block in the binary image can be seen in Figure 9, because light spot and the gray level of eyelashes are similar to those of pupil. It is found that 8-connected components method can well remove some isolated noise blocks produced by eyelashes or other elements, but the method cannot remove the light spot in pupil. The light spot in pupil is disappeared after the amendment by the line scanning method. From the amended pupil image, we can see the interfering noise decrease obviously. Thus, the calculated amount reduced significantly in Hough transform.

Under the same conditions, compared with [11] and [12] in Table 1, the speed of proposed algorithm is the fastest. They ignore the influence of noise caused by eyelashes and light spot in the process of Hough transform in [11] and [12], but this algorithm obtain a better quality pupil before locating pupil by Hough transform. In the process of segment a high quality pupil region, we remove most of these noise caused by eyelashes and light spot, consequently, it will optimize the results of the Hough transform.

Table.1. Comparison of Running Time

\begin{tabular}{|c|c|c|}
\hline Pupil Image Size & Algorithm & Running Time/s \\
\hline $964 \times 1292$ & Reference[11] [12] & 0.338 \\
\hline $964 \times 1292$ & Proposed algorithm & 0.281 \\
\hline
\end{tabular}

\section{Conclusion}

In this paper, based on characteristics of the operator's ocular movement and vision in micro operation, we propose an algorithm of pupil segmentation and localization for automated micro operation. This algorithm exhibits several advantages.

1) Rapidly segmenting high quality pupil image before locating the pupil center, reducing unnecessary vote calculation for interference points in Hough transform.

2) Effectively wiping off the interference of eyebrows and the low gradation region of image by segmenting the specific interest region. And it also dramatically cut down the processed image region to increase calculating speed.

3) Greatly reducing the subsequent processing pixels by using down sampling method 
without affecting the pupil localization accuracy.

The experimental results show that the proposed algorithm can rapidly locate the pupil center. And the proposed algorithm can meet the speediness request of micro operation.

\section{Acknowledgement}

In this paper, the research was sponsored by the National Nature Science Foundation of China (Project No. 61373110 and Project No.61272196) and Wuhan Science and Technology Research Projects (Project No. 2014010101010005).

\section{References}

[1] X. B. Pu , Y. X. Wang, H. P. Deng, and W. Li, The classified pupil localization algorithm of line-of-sight tracking system [J]. Acta Aeronautica et Astronautica Sinica. 2012: 33: 1052-1059.

[2] J. Chen, S. H. Zheng, L. Pan, and L. Yu, Fast pupil segmentation algorithm based on image feature [J]. Journal of Electronic Measurement and Instrumentation. 2014: 28:203-210.

[3] H. J. Yan, and Y. L. Zhang, Research on detecting algorithm of pupil eigenvalues in medical eye image [J]. Computer Engineering and Design. 2010: 31: 5124-5127.

[4] D. H. Li, and J. P. Derrick, Starburst: A hybrid algorithm for video-based eye tracking combining feature-based and model-based approaches. Computer Vision and Pattern Recognition-Workshops, 2005. CVPR Workshops. IEEE Computer Society Conference on. IEEE. 2005: 79-79.

[5] W. C. Zhang, B. Li, H. P. Deng, W. M. Tan, and Z. Q. Zhuang, Distorted pupil localization in eye tracking [J]. Journal of Electronics and Information Technology. 2010: 32: 416-421.

[6] C. Y. Wang, and X. Y. Shi, Research on the method of pupil localization based on videonystagmograph [J]. Computer and Digital Engineering. 2011: 39: 128-130.

[7] X. M. Wang, P. Yang, and Z. L. Wang, Pupil localization for multi-view eyeballs [J]. Journal of Computer-Aided Design and Computer Graphics. 2011: 23: 1427-1432.

[8] C. T. Sun, Z. G. Zhou, X. D. Liu, S. L. Lan, Improved iris location algorithm [J]. MINI-MICRO SYSTEMS. 2006: 27: 1335-1337.

[9] A. Liaghatdar, K. Kangarloo, and F. Farokhi, Pupil localizing in video images the first step toward eye monitoring. Multimedia Technology (ICMT), 2011 International Conference on. IEEE. 2011: 3163-3166.

[10]R. P. Wildes, "Iris Recognition: An Emerging Biometric Technology," Proceedings of IEEE, Vol. 85, pp. 1348-1363, 1997.

[11] Z. Q. Zhang, Q. L. Mengke, and L. M. Chao, Pupil radius measurement based on threshold segmentation and Hough transform algorithm [J]. Journal of Inner Mongolia Agricultural University. 2013: 34: 145-148.

[12] S. Q. Wang, Y. J. Zhao, and S. Q. Zhang, Application of hough algorithm in eye tracking and targeting [J]. Manufacturing Automation. 2013: 31: 73-74. 